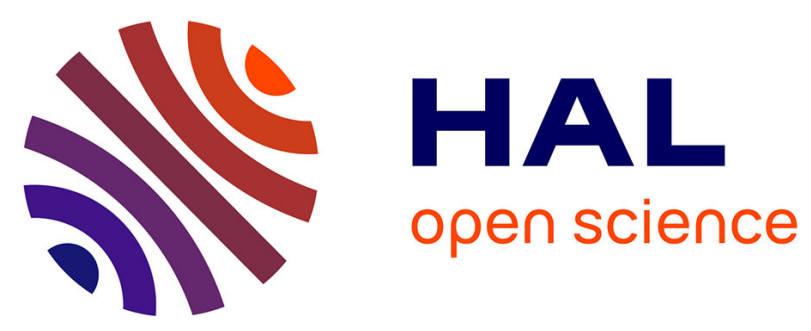

\title{
Conséquences d'une injection tunnel Fowler-Nordheim dans des structures MOS à oxyde mince
}

\author{
B. Balland, C. Plossu, S. Bardy, P. Pinard
}

\section{To cite this version:}

B. Balland, C. Plossu, S. Bardy, P. Pinard. Conséquences d'une injection tunnel Fowler-Nordheim dans des structures MOS à oxyde mince. Revue de Physique Appliquée, 1985, 20 (4), pp.225-234. 10.1051/rphysap:01985002004022500 . jpa-00245327

\section{HAL Id: jpa-00245327 https://hal.science/jpa-00245327}

Submitted on 1 Jan 1985

HAL is a multi-disciplinary open access archive for the deposit and dissemination of scientific research documents, whether they are published or not. The documents may come from teaching and research institutions in France or abroad, or from public or private research centers.
L'archive ouverte pluridisciplinaire HAL, est destinée au dépôt et à la diffusion de documents scientifiques de niveau recherche, publiés ou non, émanant des établissements d'enseignement et de recherche français ou étrangers, des laboratoires publics ou privés. 


\title{
Conséquences d'une injection tunnel Fowler-Nordheim dans des structures MOS à oxyde mince
}

\author{
B. Balland, C. Plossu, S. Bardy et P. Pinard \\ Laboratoire de Physique de la Matière (*), Institut National des Sciences Appliquées de Lyon, \\ 20, avenue A. Einstein, 69621 Villeurbanne Cedex, France
}

(Reçu le 16 mai 1984, révisé les 15 novembre 1984 et 17 janvier 1985, accepté le 31 janvier 1985)

\begin{abstract}
Résumé. - Le comportement de structures MOS à oxyde mince et à grille Si-Poly ou Al, soumises à une injection de porteurs dans $\mathrm{SiO}_{2}$ par mécanisme tunnel Fowler-Nordheim, a été étudié. Les centres de capture initialement présents dans la couche isolante de la grille ont été caractérisés par leur section efficace, leur localisation spatiale et leur concentration. Leur remplissage est indépendant de la polarité de la tension appliquée. De plus, nous avons montré qu'il y a dégradation progressive des deux interfaces délimitant la couche de $\mathrm{SiO}_{2}$. Celle-ci correspond à une génération de nouveaux sites électroniques qui ne sont pas de simples états d'interface, mais apparaissent comme des pièges induits dans $\mathrm{SiO}_{2}$. Leur taux de création et leur densité dépendent de la technologie d'élaboration. Leur génération joue un rôle déterminant quant au mécanisme de fiabilité de tels dispositifs.
\end{abstract}

\begin{abstract}
The behaviour of thin gate oxide MOS structures with Poly-Si or Al gate, subjected to a tunnelling Fowler-Nordheim injection, has been analysed. Native capture centres in the bulk of $\mathrm{SiO}_{2}$ have been characterized by their capture cross section, their density and their centroid in $\mathrm{SiO}_{2}$. The trapping is independent of the gate bias polarity during injection. The other aging mechanism is interface degradation by generation of new centres in the dioxide. This phenomenon has been analysed in correlation with the elaboration process of devices. The aging of MOS structures under electrical injection is essentially controlled by this mechanism.
\end{abstract}

\section{Introduction.}

Le piégeage-dépiégeage des porteurs dans les couches épaisses (plus de $100 \mathrm{~nm}$ ) d'oxyde thermique, constituant l'isolant de grille dans les structures MOS, a été fréquemment étudié par diverses techniques $[1,2]$. Récemment, on a constaté [3-6] que si de tels composants ont subi une injection avalanche d'électrons chauds, deux types de phénomènes sont à l'origine de la dégradation : (i) Il y a remplissage des pièges en volume dans $\mathrm{SiO}_{2}$ [4]. (ii) Il se forme une charge d'espace à l'interface $\mathrm{Si}_{-} \mathrm{SiO}_{2}$ [5], en corrélation avec la création d'états de surface lents et rapides [6,7].

Par contre, les études concernant les éléments MOS à oxyde mince $(<20 \mathrm{~nm})$ sont beaucoup moins fréquentes [8-10], bien que de telles couches isolantes soient de plus en plus souvent présentes dans les dispositifs de dimensions réduites (MOSFET, structures mémoires non volatiles à grille flottante...). Malgré le développement de la technologie, la stabilité des

(*) Laboratoire associé au C.N.R.S., LA 358. caractéristiques électriques de tels composants à oxyde mince reste aléatoire $[11,12]$. Elle dépend de divers paramètres qui définissent le substrat (préparation de la surface, dopage), du contact de grille (aluminium ou silicium polycristallin) et de la couche d'oxyde (épaisseur, méthode d'obtention...). Il est donc très important d'étudier les divers mécanismes de dégradation dans les structures MOS à oxyde mince s'établissant pendant toute contrainte d'injection, car une meilleure compréhension devrait conduire à une amélioration de leur stabilité diélectrique et de leur tenue au claquage intrinsèque.

Nous décrivons ici une procédure nouvelle permettant de suivre de manière continue, en cours même d'injection tunnel Fowler-Nordheim, l'évolution de telles structures $\left(\mathrm{Al}-\mathrm{SiO}_{2}-\mathrm{Si}\right.$ ou $\mathrm{Si}$-Poly$\mathrm{SiO}_{2}-\mathrm{Si}$ ) quand elles sont soumises à une contrainte permanente à courant d'injection constant. Cette méthode nous a permis [13, 14] d'établir l'existence d'un phénomène de création de pièges dans $\mathrm{SiO}_{2}$ par la contrainte (ce fait expérimental a été confirmé très récemment par S. Horiguchi et al. [15]). Elle 
consiste à exploiter la réponse des structures MOS, lors d'un brusque changement du sens d'injection. De par son principe, elle montre l'importance du phénomène dans les dispositifs mémoires soumis à de tels cycles. Les paramètres spécifiques de ces centres induits par la contrainte ont été déterminés (localisation, vitesse de génération, densité). Leur génération contribue, comme les autres mécanismes (piégeage par les centres pré-existants ou natifs [3,4], génération d'états de surface à l'interface $\mathrm{Si}_{-} \mathrm{SiO}_{2}[4-6]$ ), à la dégradation des caractéristiques électriques des dispositifs.

Après avoir indiqué la procédure expérimentale suivie, nous présentons quelques résultats obtenus. Les influences de la concentration, de la nature du dopage dans le substrat et dans la grille Si-Poly, de l'épaisseur d'oxyde sur le phénomène de création de nouveaux pièges sont ensuite brièvement discutés.

\section{Procédure expérimentale.}

2.1 Elaboration Des STRUCtures MOS. - Les structures MOS à oxyde mince (épaisseur $t_{\mathrm{nx}}$ entre $6 \mathrm{~nm}$ et $20 \mathrm{~nm}$ ) ont été réalisées sur silicium monocristallin d'orientation $\langle 100\rangle$ dopé phosphore $\left(10^{15}\right.$. $10^{17} \mathrm{~cm}^{-3}$ ). L'oxyde de champ permettant la réalisation du contact est de grande épaisseur (plus de $100 \mathrm{~nm}$ ); il est obtenu par oxydation locale avant la croissance de l'oxyde tunnel de grille. Plusieurs procédés d'oxydation thermique ont été utilisés (avec ou sans $\mathrm{H}_{2} \mathrm{O}$, ou $\mathrm{HCl}$, en pourcentage variable). Le matériau de grille est soit de l'aluminium, soit du Si-Poly. Ce dernier, déposé par LPCV sous une épaisseur de 450 à $550 \mathrm{~nm}$, est fortement dopé As ou $\mathrm{P}\left(5 \times 10^{19} \mathrm{~cm}^{-3}\right.$ ou $\left.10^{20} \mathrm{~cm}^{-3}\right)$. Sur certaines couches, un contact supérieur est réalisé par dépôt de $\mathrm{W}$ ou métallisation par canon à électrons avec $\mathrm{Al} / \mathrm{Si} / \mathrm{Ti}$ et photolithogravure. Les structures subissent un recuit thermique à $450^{\circ} \mathrm{C}$ pendant $30 \mathrm{~min}$ (n'ayant pas observé de différences significatives entre ces deux technologies, nous ne les distinguerons pas). Quant à la grille en $\mathrm{Al}$, elle est réalisée par métallisation sous vide immédiatement après oxydation suivie d'un traitement thermique $\left(100^{\circ} \mathrm{C}\right.$ pendant 30 min dans le " Forming gaz »).

Nous procédons d'abord à une caractérisation électrique des diverses structures en utilisant les méthodes classiques [16]. La présence éventuelle d'ions mobiles (néfaste pour les mesures ultérieures) a été contrôlée à partir de l'évolution de la capacité de la structure soumise à des contraintes champtempérature [16]. Les éléments MOS retenus présentent une densité d'ions mobiles inférieure à $2 \times 10^{10} \mathrm{~cm}^{-2}$. La qualité initiale de l'interface est évaluée soit par la méthode quasi statique (capacité en très basse fréquence), soit par la dispersion d'admittance $(C(V, \omega)$ et $G(V, \omega)$ [16]). Elle est considérée comme satisfaisante si la densité d'états de surface $N_{\mathrm{ss}}$ est inférieure à $5 \times 10^{10} \mathrm{~cm}^{-2}$. De plus, pour éviter les claquages destructifs aléatoires dus à la présence d'inhomogénéités dans l'oxyde, l'aire des éléments MOS est petite $\left(6,25 \times 10^{4} \mu \mathrm{m}^{2}\right)$. Les structures retenues doivent présenter une bonne rigidité diélectrique, le champ de claquage devant être supérieur à $10^{7} \mathrm{~V} / \mathrm{cm}$.

2.2 Evolution SOUS CONTRAINTE D'INJECTION F.N. L'injection d'électrons par mécanisme tunnel FowlerNordheim peut être réalisée à partir soit de la grille, soit du substrat (mode - ou mode + ) selon que la polarisation $V_{\mathrm{G}}$ est négative ou positive, le substrat étant de type $\mathrm{N}$. La conséquence d'une telle injection est un piégeage des porteurs dans l'oxyde [1], d'où la formation d'une charge d'espace dans $\mathrm{SiO}_{2}$ ou à proximité des interfaces. Il en résulte alors une modification de la hauteur et du profil de la barrière de potentiel à l'interface $\mathrm{SiO}_{2}$-cathode [16], et une évolution de la distribution du champ dans l'oxyde. Cela se traduit par une dérive des courbes $I_{\mathrm{F} . \mathbf{N}}-V_{\mathrm{G}}$ le long de l'axe des tensions [9]. Pour maintenir constante la densité de courant tunnel, il faut neutraliser la variation du champ à la cathode, c'est-à-dire réajuster la tension de grille $V_{\mathrm{G}}$, de $\Delta V_{\mathrm{G}}$. On peut exploiter cette translation des courbes $I_{\mathrm{F} . \mathrm{N}}-V_{\mathrm{G}}$ pour accéder à certains paramètres des pièges en volume (densité, localisation). Selon un modèle électrostatique classique $[9,16]$, en écrivant, pour les deux modes d'injection, la condition de champ constant à l'interface $\mathrm{SiO}_{2}$-cathode, nous obtenons :

$\Delta V_{\mathrm{G}}^{+}=-\frac{\bar{x}}{\varepsilon_{\mathrm{ox}}} Q_{\mathrm{ox}} \quad$ et $\quad \Delta V_{\mathrm{G}}^{-}=\frac{t_{\mathrm{ox}}}{\varepsilon_{\mathrm{ox}}}\left(1-\frac{\bar{x}}{t_{\mathrm{ox}}}\right) Q_{\mathrm{ox}}$

où $\varepsilon_{\mathrm{ox}}, t_{\mathrm{ox}}, Q_{\mathrm{ox}}$ et $\bar{x}$ sont respectivement la permittivité et l'épaisseur de l'oxyde, la densité et l'abscisse du barycentre des charges piégées. La connaissance de $\Delta V_{\mathrm{G}}$ en mode $(+)$ et $(-)$ permet de déterminer $Q_{\mathrm{ox}}$ et $\bar{x}$ :

$Q_{\mathrm{ox}}=\frac{\varepsilon_{\mathrm{ox}}}{t_{\mathrm{ox}}}\left(\Delta V_{\mathrm{G}}^{-}-\Delta V_{\mathrm{G}}^{+}\right) \quad$ et $\quad \bar{x}=t_{\mathrm{ox}}\left(1-\frac{\Delta V_{\mathrm{G}}^{-}}{\Delta V_{\mathrm{G}}^{+}}\right)^{-1}$.

Les charges piégées dans les états de surface n'ont qu'une influence négligeable sur l'intensité du courant F.N., donc sur la variation de la tension nécessaire pour maintenir l'injection à un niveau constant. En exploitant ainsi les caractéristiques $C-V_{\mathrm{G}}$ (mesure de la tension de dérive $\left.\Delta V_{\mathrm{FB}}\right), G-V_{\mathrm{G}}$ et $I_{\mathrm{F} . \mathrm{N} .}-V_{\mathrm{G}}$, on peut étudier le piégeage dans le volume de $\mathrm{SiO}_{2}$ et la dégradation de l'interface $\mathrm{Si}_{-} \mathrm{SiO}_{2}$. Mais toutes ces mesures étant réalisées après interruption de l'injection, elles ne sont pas représentatives du comportement dynamique de la structure MOS pendant la contrainte.

Afin de suivre l'évolution de celle-ci, dans des conditions plus proches de celles correspondant au fonctionnement des composants (par exemple des dispositifs mémoires), nous avons réalisé un appa- 
reillage permettant de compenser automatiquement la dérive en tension. La polarisation de grille est alors régulée $\left(V_{\mathrm{G}} \equiv V_{\mathrm{R}}\right)$ de sorte que la densité du courant d'injection reste constante (entre $10^{-7} \mathrm{~A} / \mathrm{cm}^{2}$ et $1 \mathrm{~A} / \mathrm{cm}^{2}$ ). Nous pouvons ainsi enregistrer $V_{\mathrm{R}}^{+}$ou $V_{\mathrm{R}}^{-}$ en fonction du temps, jusqu'au claquage destructif. La figure 1 représente de telles courbes d'évolution (courbes « de charge ») pour les deux modes d'injection, avec par exemple $J=0.64 \mathrm{~A} / \mathrm{cm}^{2}$. On constate qu'elles ne présentent pas de saturation; elles sont interrompues par le claquage destructif de l'élément. Leur profil n'est donc pas exponentiel, contrairement à ce que prévoit le modèle classique $[1,4]$ supposant une cinétique du premier ordre pour le remplissage des pièges pré-existants. Rappelons que cette interprétation est généralement suffisante dans le cas de l'injection avalanche dans les MOS à oxyde épais (plus de 50 à $100 \mathrm{~nm}$ ) [1-5, 16]. Mais la non-saturation apparaissant sur les courbes expérimentales montre l'insuffisance de cette hypothèse. On pourrait envisager la présence d'un ensemble de pièges, certains d'entre eux ayant des sections efficaces de capture $\sigma_{i}$ très faibles. $V_{\mathrm{R}}\left(t_{\mathrm{inj}}\right)$ serait alors interprété comme une somme de termes exponentiels; mais pour expliquer correctement le profil de la courbe d'évolution, il serait nécessaire de choisir des constantes de temps $\tau_{\mathbf{i}}$ (soit $q / \sigma_{\mathrm{i}} J$ ) anormalement grandes ( $\sigma_{\mathrm{i}}$ très petite) sans signification physique.

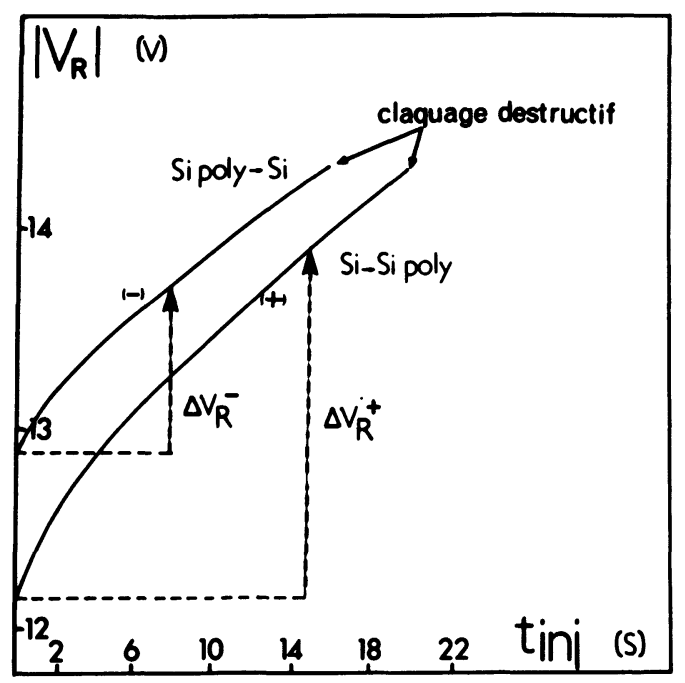

Fig. 1. - Evolution à température constante $(300 \mathrm{~K})$ de la tension de régulation $V_{\mathrm{R}}$ pendant l'injection $\mathrm{F}$.N. à courant constant $\left(J_{\text {inj }}=0,64 \mathrm{~A} / \mathrm{cm}^{2}\right)$ en mode $(+)$ et en mode $(-)$, pour des structures MOS à oxyde mince $\left(t_{\mathrm{ox}}=10 \mathrm{~nm}\right.$ et $S=62500 \mu \mathrm{m}^{2}$ ).

[Evolution at constant temperature $(300 \mathrm{~K})$ of the regulated voltage $V_{\mathrm{R}}$ during a Fowler-Nordheim constant current injection $\left(J_{\text {inj }}=0.64 \mathrm{~A} / \mathrm{cm}^{2}\right)$ for both injection modes in the case of thin oxide MOS structures $\left(t_{\mathrm{ox}}=10 \mathrm{~nm}\right.$ and $\left.S=62500 \mu \mathrm{m}^{2}\right)$.]
Nous interprétons ce comportement transitoire des structures MOS à oxyde mince, soumises à une injection tunnel F.N., en supposant la contribution de deux mécanismes de piégeage différents : (i) il y a capture des électrons par les centres natifs; (ii) il y a création de nouveaux pièges qui se remplissent très vite. La procédure expérimentale permet de séparer correctement ces deux types de centres de capture et de déterminer les paramètres de chacun; les grandeurs correspondantes sont indicées « ex » et « ind » respectivement pour les centres natifs, ou se formant pendant l'injection.

Selon le modèle brièvement présenté en annexe, nous écrivons que la variation de la tension de régulation pendant la contrainte $\left(t_{\text {inj }}=\right.$ durée de l'injection) correspond au remplissage des deux types de pièges :

$$
\Delta V_{\mathrm{R}}\left(t_{\mathrm{inj}}\right)=V_{\mathrm{R}}\left(t_{\mathrm{inj}}\right)-V_{\mathrm{R}}(0)=\Delta V_{\mathrm{R} \text { ex }}+\Delta V_{\mathrm{R} \text { ind }}
$$

soit d'après la relation (A.9) :

$$
\begin{aligned}
\Delta V_{\mathbf{R}}\left(t_{\text {in }}\right)=\frac{q}{\varepsilon_{\text {ox }}} \bar{x}_{\text {ex }} & N_{\text {ex }}\left\{1-\exp \left(\frac{\sigma_{\text {ex }} J}{q} t_{\text {inj }}\right)\right\}+ \\
+ & \frac{\bar{x}_{\text {ind }} r J t_{\text {inj }}}{\varepsilon_{\text {ox }}}-\frac{\bar{x}_{\text {ind }} r 1 / \sigma_{\text {ind }}}{\varepsilon_{\text {ox }}} \\
& \left.\times\left\{1-\exp \left(-\frac{\sigma_{\text {ind }} J}{q}\right) t_{\text {inj }}\right)\right\}
\end{aligned}
$$

$r$ représente le taux de création des nouveaux centres :

$$
r=\frac{q}{J} \cdot \frac{\partial N_{\mathrm{ind}}}{\partial t_{\mathrm{inj}}}=\frac{\partial Q_{\mathrm{ind}}}{J \partial t_{\mathrm{inj}}}
$$

$\bar{x}_{\text {ex }}, \bar{x}_{\text {ind }}, N_{\text {ex }}, N_{\text {ind }}, \sigma_{\text {ex }}$ et $\sigma_{\text {ind }}$ sont respectivement les abscisses des barycentres, les densités et les sections efficaces de capture des centres natifs (ex) et induits (ind). $\Delta V_{\mathbf{R} \text { ex }}\left(t_{\text {inj }}\right)$, correspondant au remplissage des centres natifs, présente un profil exponentiel, avec une saturation après une durée d'injection relativement courte. $\Delta V_{\mathrm{R} \text { ind }}\left(t_{\mathrm{inj}}\right)$ comporte deux termes, le premier traduit la formation de centres et le second, leur remplissage. En décomposant ainsi la réponse $\Delta V_{\mathrm{R}}\left(t_{\mathrm{inj}}\right)$, comme le montre la figure 2 , nous obtenons effectivement une première contribution exponentielle, mais la seconde présente un profil linéaire. Nous en déduisons que le troisième terme de l'équation (4) doit être négligeable; les centres induits ont donc une section efficace de capture très grande et ils se remplissent dès leur formation. Un tel comportement pourrait être expliqué par la présence de défauts cristallographiques instables, initialement inactifs, l'injection d'électrons entraînant une évolution de leur configuration. $\Delta V_{\mathrm{R} \text { ind }}\left(t_{\mathrm{inj}}\right)$ est donc une fonction linéaire; la pente $p$ de la courbe correspond au produit $r \cdot \bar{x}_{\text {ind }}$ (rel. (4)).

Pour étudier séparément le phénomène de création de nouveaux centres et celui de remplissage des pièges natifs, nous avons procédé de la manière suivante. 


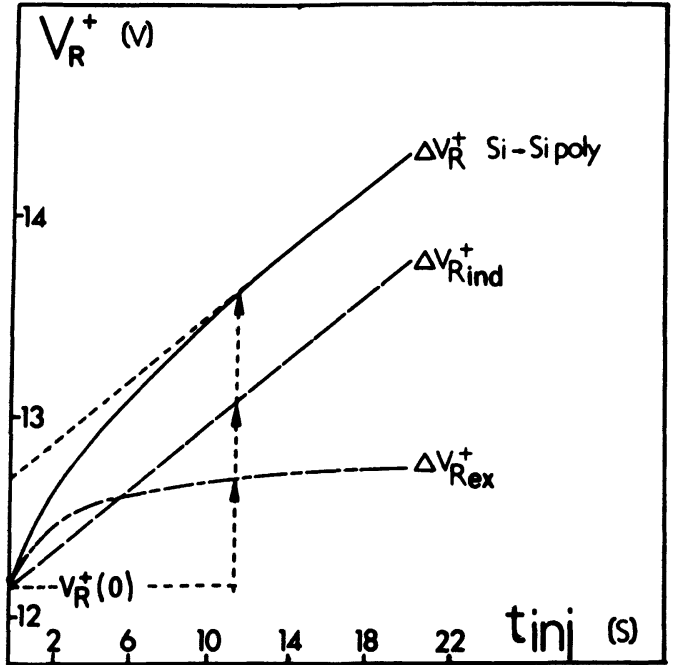

Fig. 2. - Décomposition de $\Delta V_{\mathbf{R}}^{+}$montrant la coexistence de deux mécanismes de remplissage des centres de capture dans $\mathrm{SiO}_{2}\left(\Delta V_{\mathrm{Rex}}^{+}\right.$pièges natifs et $\Delta V_{\mathrm{R} \text { ind }}^{+}$pièges formés pendant la contrainte, $\Delta V_{\mathrm{R}}^{+}=\Delta V_{\mathrm{R} \text { ex }}^{+}+\Delta V_{\mathrm{R} \text { ind }}^{+}$).

[Decomposition of $\Delta V_{R}^{+}$showing the coexistence of two traps filling mechanisms in $\mathrm{SiO}_{2}\left(\Delta V_{\mathrm{R} \text { ex }}^{+}\right.$native centres and $\Delta V_{\mathrm{R} \text { ind }}^{+}$induced traps during the stress, $\Delta V_{\mathrm{R}}^{+}=\Delta V_{\mathrm{R} \text { ex }}^{+}+$ $\left.\Delta V_{\mathrm{R} \text { ind }}^{+}\right)$.]

On analyse le comportement d'un ensemble de structures MOS appartenant à une même tranche de silicium, soumises à une injection F.N. à intensité de courant constante, mais dans des conditions différentes (Fig. 3) :

1) Pour un premier élément MOS, nous enregistrons l'évolution de $V_{\mathbf{R}}^{+}$pour une injection primaire en mode $(+)$ pendant une durée $t_{\text {inj }}^{+}=t_{1}$. A cet instant $t_{1}$, nous inversons le sens de l'injection et notons la tension de démarrage. Analysons le comportement de la structure pendant les deux phases. (i) Pendant la première, les électrons sont capturés par les centres natifs et induits. La barrière de potentiel interfaciale est alors modifiée par la présence de cette nouvelle charge piégée (abaissement de la hauteur et déplacement du sommet [16]). Le comportement de la structure sous injection est alors décrit par la relation (4) simplifiée :

$$
\begin{aligned}
& \Delta V_{\mathrm{R}}^{+}\left(t_{\mathrm{inj}}^{+}\right)=\frac{q}{\varepsilon_{\mathrm{ox}}} \bar{x}_{\mathrm{ex}} N_{\mathrm{ex}} \times \\
& \quad \times\left\{1-\exp \left(-\frac{\sigma_{\mathrm{ex}} J}{q} t_{\mathrm{inj}}^{+}\right)\right\}+\frac{\bar{x}_{\mathrm{ind}}^{+}}{\varepsilon_{\mathrm{ox}}} r^{+} J t_{\mathrm{inj}}^{+} .
\end{aligned}
$$

(ii) Lorsque le sens d'injection est inversé $\left(t_{\mathrm{inj}}=t_{1}\right)$, la dérive $\Delta V_{\mathrm{R}}^{-}\left(t_{1}\right)$ correspond à la totalité de la charge piégée dans le volume de la couche d'oxyde, c'est-àdire aux centre natifs et induits pendant la première phase :

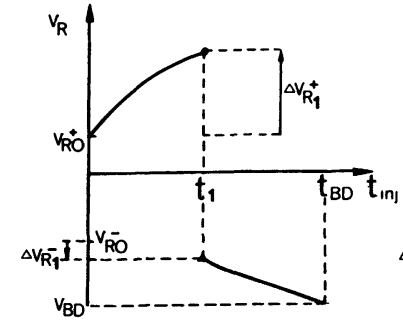

a

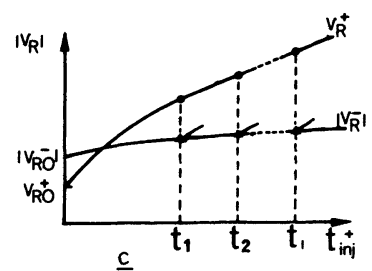

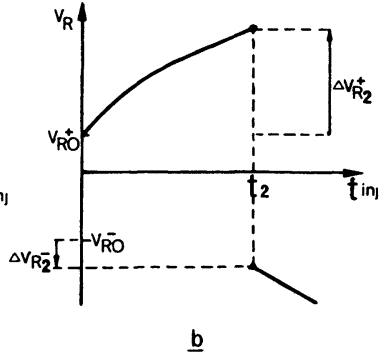

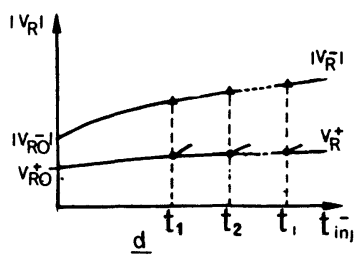

Fig. 3. - Diagrammes décrivant la procédure expérimentale adoptée pour la détermination des paramètres des pièges induits $\left(\bar{x}_{\text {ind }}\right.$ et $\left.N_{\text {ind }}\right)$; a) élément MOS1 soumis à l'injection en mode $(+)$ pendant une durée $t_{1}$ puis en mode (-) jusqu'au claquage $\left.\left(t_{\mathrm{BD}}\right) ; \mathrm{b}\right)$ élément MOS2 soumis à la même contrainte pendant la durée $t_{2} ; \mathrm{c}$ ) courbe " directe " et "inverse " dans le cas d'une injection cumulative en mode primaire $(+)$; d) injection cumulative primaire en mode $(-)$.

[Diagrams describing the experimental procedure for the determination of induced traps parameters $\left(x_{\text {ind }}\right.$ and $\left.N_{\text {ind }}\right)$; a) element MOS1 subjected to an injection in mode $(+)$ during the time $t_{1}$ then in mode (-) until breakdown $\left(t_{\mathrm{BD}}\right)$; b) element MOS2 subjected to the same stress during the time $\left.t_{2} ; \mathrm{c}\right)$ " direct " and " inverted " curves for the case of a cumulative injection in primary mode $(+)$; d) cumulative injection in primary mode $(-)$.]

$$
\Delta V_{\mathrm{R}}^{-}\left(t_{1}\right)=\frac{t_{\mathrm{ox}}}{\varepsilon_{\mathrm{ox}}}\left(1-\frac{\bar{x}_{\mathrm{ind}}^{+}}{t_{\mathrm{ox}}}\right) Q_{\mathrm{ind}}^{+}+\frac{t_{\mathrm{ox}}}{\varepsilon_{\mathrm{ox}}}\left(1-\frac{\bar{x}_{\mathrm{ex}}}{t_{\mathrm{ox}}}\right) Q_{\mathrm{ex}}
$$

avec

$$
\begin{gathered}
Q_{\mathrm{ind}}^{+}\left(t_{1}\right)=r^{+} J t_{1} \\
\text { et } \quad Q_{\mathrm{ex}}\left(t_{1}\right)=q N_{\mathrm{ex}}\left\{1-\exp \left(-\frac{\sigma_{\mathrm{ex}} J}{q} t_{1}\right)\right\} .
\end{gathered}
$$

2) La même contrainte est appliquée à un deuxième élément MOS de la même tranche de $\mathrm{Si}$, et présentant initialement les mêmes caractéristiques électriques. La durée de l'injection est alors $t_{\mathrm{inj}}^{+}=t_{2}>t_{1}$. L'évolution est analysée comme précédemment.

En procédant ainsi, pour un grand nombre de structures MOS d'une même tranche de $\mathrm{Si}$, il est possible de relever point par point $\Delta V_{\mathrm{R}}^{-}$en fonction de $t_{\mathrm{inj}}^{+}$. La courbe ainsi obtenue est représentée sur la figure 4. Lorsque $t_{\mathrm{inj}}^{+}$est suffisamment long, il y a saturation des centres natifs et donc extinction du régime transitoire correspondant à leur remplissage. Les équations 


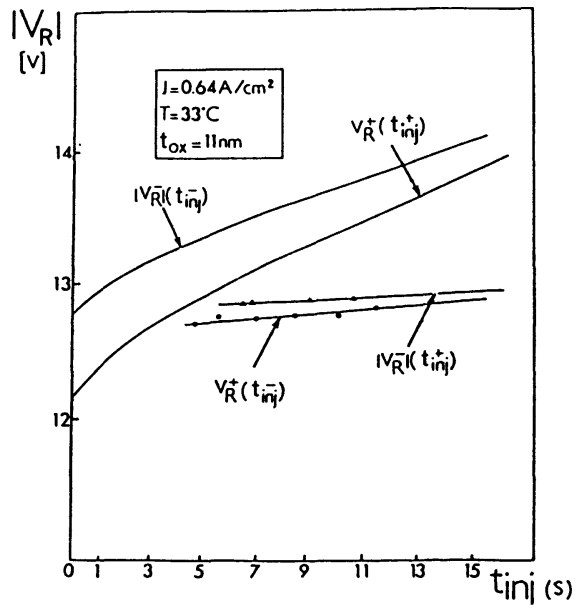

Fig. 4. - Courbes expérimentales permettant la détermination des paramètres des centres induits dans les deux modes d'injection $\left(T=300 \mathrm{~K}, t_{\mathrm{ox}}=11 \mathrm{~nm}, J_{\mathrm{inj}}=0,64 \mathrm{~A} / \mathrm{cm}^{2}\right)$.

[Experimental curves leading to the determination of induced traps parameters for both injection modes $\left(T=300 \mathrm{~K}, t_{\mathrm{ox}}=11 \mathrm{~nm}, J_{\mathrm{inj}}=0.64 \mathrm{~A} / \mathrm{cm}^{2}\right)$.]

(6) et (7) se réduisent à :

$$
\Delta V_{\mathrm{R}}^{+}\left(t_{\mathrm{inj}}^{+}\right) \# p^{+} t_{\mathrm{inj}}^{+} \text {et } \Delta V_{\mathrm{R}}^{-}\left(t_{\mathrm{inj}}^{+}\right) \# p^{-} t_{\mathrm{inj}}^{+}
$$

avec

$$
p^{+}=\frac{r^{+} \bar{x}_{\text {ind }}^{+}}{\varepsilon_{\mathrm{ox}}} \text { et } p^{-}=r^{+} \cdot \frac{t_{\mathrm{ox}}}{\varepsilon_{\mathrm{ox}}}\left(1-\frac{\bar{x}_{\mathrm{ind}}^{+}}{t_{\mathrm{ox}}}\right) \mathrm{J} .
$$

La connaissance des pentes $p^{+}$et $p^{-}$de $\Delta V_{\mathrm{R}}^{+}\left(t_{\mathrm{inj}}^{+}\right)$et $\Delta V_{\mathrm{R}}^{-}\left(t_{\mathrm{inj}}^{+}\right)$permet de déterminer séparément $r^{+}$et $\bar{x}_{\text {ind }}^{+}$, c'est-à-dire la vitesse de génération et la position du barycentre des pièges induits pendant l'injection F.N., dans le sens substrat (type $\mathrm{N}$ ) $\rightarrow$ grille (injection en mode + ).

La même procédure expérimentale dans le cas d'une injection primaire en mode $(-)$ conduit au calcul de $r^{-}$et $\bar{x}_{\text {ind }}^{-}$, paramètres spécifiques des centres induits lors d'une injection à partir de la grille.

Les centres natifs sont caractérisés en exploitant les parties exponentielles de $\Delta V_{\mathbf{R}}^{+}\left(t_{\mathrm{inj}}^{+}\right)$et $\Delta V_{\mathbf{R}}^{-}\left(t_{\mathrm{inj}}^{-}\right)$ pour accéder à $\bar{x}_{\mathrm{ex}}, \sigma_{\mathrm{ex}}$ et $N_{\mathrm{ex}}$ selon la méthode classique $[1,2]$. On représente graphiquement l'efficacité effective de piégeage en fonction de la durée d'injection (Fig. 5)

$$
\begin{array}{r}
\eta_{\mathrm{eff}}=\sum_{i}\left(N_{\mathrm{ex}} \cdot \frac{\bar{x}_{\mathrm{ex}}}{t_{\mathrm{ox}}} \cdot \sigma_{\mathrm{ex}}\right)_{i} \exp \left(-\frac{\sigma_{\mathrm{ex} i} J}{q} t_{\mathrm{inj}}\right)= \\
=\frac{C_{\mathrm{ox}}}{J} \frac{\mathrm{d} \Delta V_{\mathrm{Rex}}}{\mathrm{d} t_{\mathrm{inj}}} .
\end{array}
$$

Le tableau I résume l'ensemble des résultats obtenus pour les centres natifs et induits.

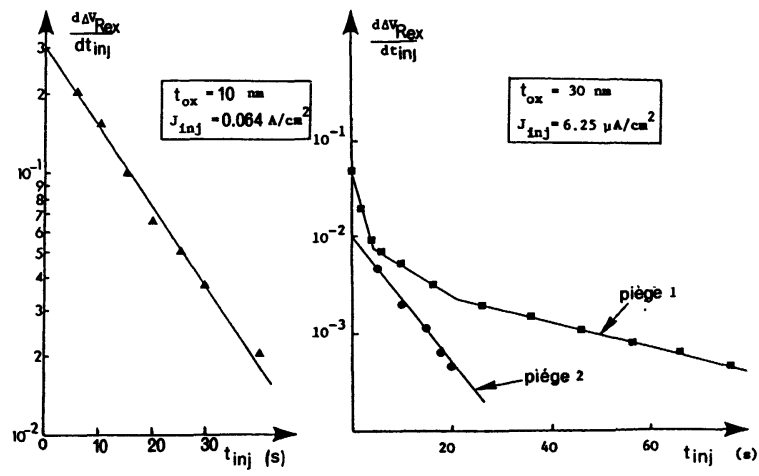

Fig. 5. - Détermination des caractéristiques des centres de capture natifs pour deux séries d'échantillons.

[Determination of native traps parameters for two types of samples.]

Tableau I. - Paramètres caractéristiques des pièges natifs et induits pour des structures à grille Si-Poly dopé $\mathrm{P}$ ou As $\left(J_{\mathrm{inj}}=0,64 \mathrm{~A} / \mathrm{cm}^{2}, t_{\mathrm{ox}}=10 \mathrm{~nm}, T=\right.$ $300 \mathrm{~K})$.

\begin{tabular}{|c|c|c|c|c|c|c|c|}
\hline & \multicolumn{3}{|c|}{ PIEGES NATIFS } & \multicolumn{4}{|c|}{ PIEGES INDUITS } \\
\hline & $\begin{array}{l}\Gamma_{\mathrm{ex}} \\
\mathrm{cm}^{2}\end{array}$ & $\underset{\mathrm{Nex}}{\mathrm{N}_{\mathrm{ex}}-2}$ & $\frac{\overline{\bar{x}}_{\text {ex }}}{t_{\text {tox }}}$ & $\mathrm{r}^{+}$ & $\begin{array}{l}t_{o x}-\bar{x}_{\text {ind }}^{+} \\
n m\end{array}$ & $r^{-}$ & $\begin{array}{l}\bar{x}_{\text {ind }} \\
n m\end{array}$ \\
\hline $\begin{array}{c}\text { GRILLE } \\
\text { Si Paly dopé } P\end{array}$ & $4.7 \times 10^{-20}$ & $1.7 \times 10^{12}$ & 0.4 & $5 \times 10^{-8}$ & 1.5 & $4.3 \times 10^{-8}$ & 2.3 \\
\hline $\begin{array}{c}\text { GRILLE } \\
\text { SiPoly dopé As }\end{array}$ & $5.7 \times 10^{-20}$ & $1.7 \times 10^{12}$ & 0.4 & $3.2 \times 10^{-8}$ & 1.5 & $4 \times 10^{-8}$ & 2.3 \\
\hline
\end{tabular}

[Native and induced traps parameters for $\mathrm{P}$ or As doped Poly-Si gate structures $\left(J_{\text {inj }}=0.64 \mathrm{~A} / \mathrm{cm}^{2}\right.$, $\left.t_{\mathrm{ox}}=10 \mathrm{~nm}, T=300 \mathrm{~K}\right)$.]

\section{Résultats.}

Dès qu'un flux de porteurs injectés par mécanisme tunnel Fowler-Nordheim franchit l'interface cathode$\mathrm{SiO}_{2}$, nous constatons que la couche d'oxyde mince est le siège de trois types de mécanismes conduisant à la dégradation des structures MOS : (1) piégeage dans les centres natifs, (2) création de nouveaux centres induits, (3) génération d'états d'interface (lents et rapides). Nos résultats ont confirmé les points (1) et (3) déjà analysés par divers auteurs $[6,10,11]$. Par contre le mécanisme (2) que nous avons mis en évidence et précisé, devient particulièrement important lorsque l'épaisseur de la couche d'oxyde diminue.

Pour séparer les divers mécanismes nous avons exploité la variation de $V_{\mathrm{R}}$ sous injection et la dérive de la tension de bandes plates, ainsi que l'évolution des profils de $C-V$ et $G-V$ entre chaque séquence d'injection.

1) Les sites préexistants que nous avons caractérisés 
dans les structures étudiées ont une très faible section efficace de capture ( $\sigma_{\mathrm{ex}}$ de l'ordre de $5 \times 10^{-20} \mathrm{~cm}^{2}$ ). Elle augmente avec le champ électrique et la température. On peut donc considérer qu'il s'agit de centres coulombiens répulsifs [1]. Leur densité se situe, selon les tranches de silicium entre $10^{11}$ et $10^{13} \mathrm{~cm}^{-2}$. Leur barycentre se trouve à $4 \mathrm{~nm}$ de l'interface Si$\mathrm{SiO}_{2}$. Dans le cas des structures $\mathrm{Si}-\mathrm{Poly}-\mathrm{SiO}_{2}-\mathrm{Si}$, les caractéristiques des centres natifs sont indépendantes de l'impureté de dopage du Si-Poly. Nous avons constaté que leur densité augmente avec la température d'oxydation et de recuit sous atmosphère inerte. L'oxydation en présence de $\mathrm{HCl}$ tend à accroître celle-ci. Ces observations, qui sont purement qualitatives, suggèrent que ces centres natifs se forment lors des opérations nécessitant des températures élevées (oxydation, recuit). Ce sont des défauts intrinsèques au système $\mathrm{Si}_{-} \mathrm{SiO}_{2}$. Il pourrait s'agir d'atomes de $\mathrm{Si}$ excédentaires (observation par microscopie électronique [7]) résultant d'un processus chimique selon lequel il se constituerait $\mathrm{SiO}$ 《gazeux » à l'interface $\mathrm{Si}-\mathrm{SiO}_{2}$, diffusant vers le volume de l'oxyde. Précisons que la présence de $\mathrm{HCl}$ semble accroître la densité d'inclusion [18].

2) Il y a création et remplissage instantané de centres induits pendant la contrainte. Ces pièges se localisent près de l'interface cathodique. Leur taux de formation est constant pendant toute la durée de la contrainte (à température fixe); mais le processus de génération est activé thermiquement.

Ainsi, lorsque l'injection se produit dans le sens $\mathrm{Si} \rightarrow$ Si-Poly (mode + ), le taux de création $r^{+}$est de $5 \times 10^{-8}$ (pièges par électron injecté) à $300 \mathrm{~K}$. Le barycentre se situe à environ $15 \mathrm{~nm}$ de l'interface Si$\mathrm{SiO}_{2}$. En injection dans le sens Si-Poly $\rightarrow \mathrm{Si}$ (mode - ), ces paramètres deviennent $r^{-}=4 \times 10^{-8}$ et $\bar{x}_{\text {ind }}=$ $2.3 \mathrm{~nm}$, distance par rapport à l'interface Si-Poly$\mathrm{SiO}_{2}$. Cependant il faut noter que ces valeurs ne sont obtenues qu'avec une incertitude assez grande (environ $10 \%$ ). Précisons que le dopage du substrat est assez faible pour qu'il soit possible d'éliminer la possibilité d'avalanche dans $\mathrm{Si}$ (pour $N_{\mathrm{D}}<8 \times$ $10^{16} \mathrm{~cm}^{-3}$, le potentiel d'avalanche est supérieur à 11 volts), dans les conditions de polarisation établies au cours de ces expériences.

La concentration de ces pièges augmente linéairement en fonction du nombre d'électrons injectés, jusqu'au claquage destructif de l'oxyde (par exemple, lorsqu'elle atteint $4 \times 10^{18} \mathrm{~cm}^{-3}$ ). La charge négative induite crée une variation de tension de bandes plates de l'ordre de $2 \mathrm{~V}$ avant le claquage. Le taux de création augmente rapidement lorsque la température croît (Fig. 6). Le processus est donc thermiquement activé :

$$
r=r_{0} \exp \left(-\frac{E_{\mathrm{A}}}{k T}\right)
$$

avec $E_{\mathrm{A}}$ égale à $0,195 \mathrm{eV}$ en mode $(+)$ et $0,150 \mathrm{eV}$ en mode $(-)$.

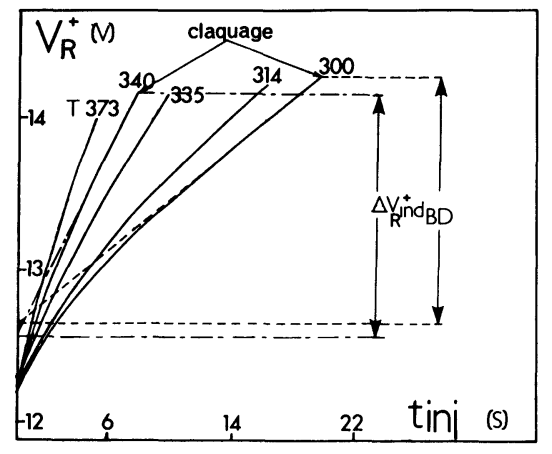

a)

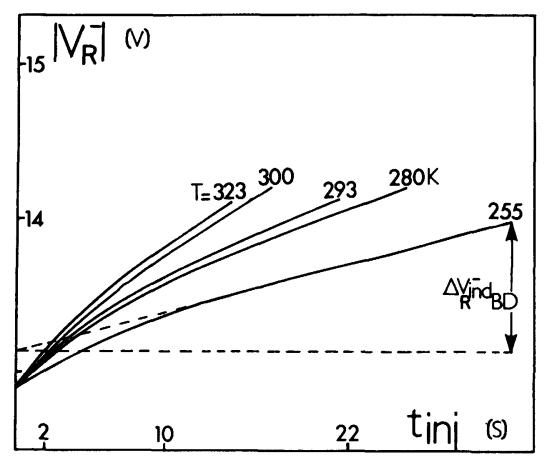

b)

Fig. 6. - Evolution de la tension de régulation en fonction du temps d'injection pour différentes températures $T$; a) injection en mode $(+)$ (sens $\mathrm{Si} \rightarrow \mathrm{Si}$-Poly); b) injection en mode $(-)$ (sens Si-Poly $\rightarrow \mathrm{Si}$ ).

[Evolution of the regulated voltage versus injection time at various temperatures $T$; a) injection in mode $(+)$ $(\mathrm{Si} \rightarrow$ Poly-Si); b) injection in mode $(-)$ (Poly-Si $\rightarrow \mathrm{Si})$.]

La nature physico-chimique des centres induits est actuellement inconnue, ainsi que leur mécanisme de formation. Il pourrait s'agir de pièges associés à des défauts intrinsèques tels que des liaisons $\mathrm{Si}-\mathrm{O}$ contraintes ou brisées par le champ électrique et/ou les porteurs très énergétiques [15].

Le claquage destructif dans les structures MOS à oxyde mince apparaît initié par la formation des centres induits. Il ne s'établit que si leur densité est suffisamment grande; notamment à proximité de l'interface $\mathrm{Si}_{-} \mathrm{SiO}_{2}$. Comme le montre la figure $6 \mathrm{a}$, la durée d'injection avant claquage est corrélée à $N_{\text {ind }}\left(t_{\mathrm{inj}}\right)$. Notons que la valeur limite atteinte par $N_{\text {ind }}$ ne dépend pas de la température. Cela suggère que le paramètre déterminant est $N_{\text {ind }}$ plutôt que le taux de création $r$.

3) Les contraintes d'injection produisent une dégradation de l'interface $\mathrm{Si}_{-} \mathrm{SiO}_{2}[6]$; ce phénomène a déjà été étudié dans des structures MOS à oxyde épais [3-7] ou mince [10]. Dans le cas des éléments étudiés, nous avons observé la formation d'états de surface. (i) Certains, qualifiés de rapides, présentent une section efficace de $10^{-15} \mathrm{~cm}^{2}$, indépendamment du sens de l'injection. Par contre leur densité $N_{\text {ss }}$ est fonction du nombre de porteurs injectés $N_{\text {inj }}$, comme le montre la figure 7. La loi est de la forme $N_{\mathrm{ss}}=R\left(N_{\mathrm{inj}}\right)^{\alpha}$. 


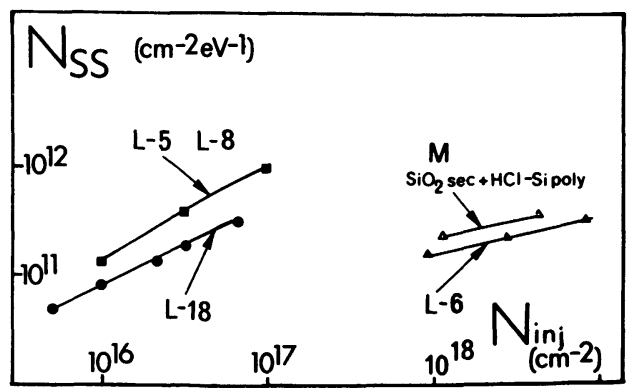

Fig. 7. - Comparaison de l'évolution de $N_{\mathrm{ss}}$ avec la densité de charge injectée pour les divers types d'échantillons.

[Comparison of the evolution of $N_{\mathrm{ss}}$ with the injected charge density for the various types of samples.]

L'exposant $\alpha$ dépend du procédé d'élaboration. Pour les structures à grille Si-Poly, il est de l'ordre de 0.25 ; par contre pour celles à grille $\mathrm{Al}$, il atteint 0.75 . Le facteur $R$ ne dépend ni de la température, ni du champ électrique. (ii) D'autres états, qualifiés d'états lents, sont également créés près de l'interface [6]. Ils apparaissent comme des centres de type donneur et sont responsables d'une instabilité des courbes $C-V$, après injection. On observe une dérive négative correspondant à la formation d'une charge positive dans $\mathrm{SiO}_{2}$ près de l'interface $\mathrm{Si}-\mathrm{SiO}_{2}$.

\section{Discussion.}

En procédant sur un grand nombre d'éléments, nous avons pu obtenir des résultats statistiques et en déduire les remarques suivantes.

1) Le barycentre des pièges induits se situe très près de l'interface injectante (à moins de $3 \mathrm{~nm}$ ). L'écart entre $r^{+}$et $r^{-}$ne permet pas de comparer les mécanismes de création aux deux interfaces. En ce qui concerne l'influence de la technologie, nous constatons (Fig. 8) que le phénomène de piégeage par les

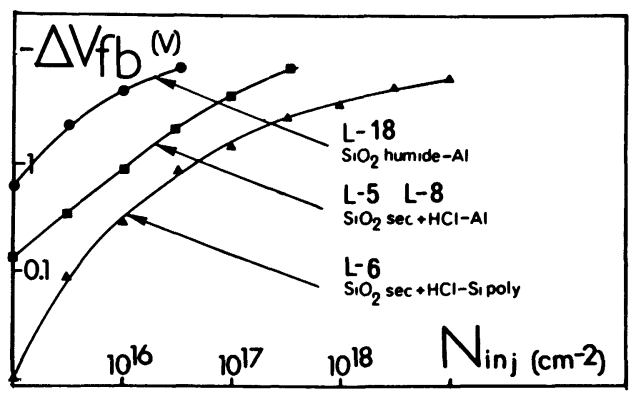

Fig. 8. - Courbes d'évolution de $\Delta V_{\mathrm{FB}}$ due à la charge positive interfaciale en fonction de la densité de charge injectée pour les différents types d'échantillons.

[Evolution curves of $\Delta V_{\mathrm{FB}}$ due to the positive interfacial charge as a function of injected charge density for the different types of MOS structures.] centres induits dépend de l'épaisseur de $\mathrm{SiO}_{2}$, de la nature et de la concentration des impuretés de dopage dans la grille Si-Poly (éventuellement dans le substrat). Le taux de génération diminue quand $t_{\mathrm{ox}}$ augmente (pour $5 \mathrm{~nm}<t_{\mathrm{ox}}<15 \mathrm{~nm}$ ). En comparant les densités de pièges et les taux de génération dans les structures à oxyde réalisé sur des substrats de dopage As ou $P$, ou avec des grilles Si-Poly contenant ces impuretés, nous avons constaté que le phénomène de formation de nouveaux pièges est moins important en présence de As ( $r$ et $N_{\text {ind }}$ sont plus faibles). Mais le claquage de l'oxyde apparaît pour des densités de courant injecté plus petites. Notons que les structures $\mathrm{Al}_{-}-\mathrm{SiO}_{2}-\mathrm{Si}$ présentent toujours un taux de génération élevé et un claquage destructif pour de faibles flux d'injection.

2) La variation négative de la tension de bandes plates $\Delta V_{\mathrm{FB}}$ (due à la charge interfaciale) augmente plus rapidement pour les grilles $\mathrm{Al}$ que pour celles en Si-Poly (facteur multiplicatif de 10 ou 5 selon que l'oxyde contient ou non de l'eau (Fig. 8)). Notons que $\Delta V_{\mathrm{FB}}$ maximale atteinte au claquage est la même pour toutes les structures; il y a donc une corrélation entre la charge positive et le processus de claquage.

3) La variation de la densité d'états de surface rapides $\Delta N_{\mathrm{ss}}$ est corrélée à celle des charges interfaciales $\Delta N_{\mathrm{FB}}$ (Fig. 9). Une telle observation a déjà été rapportée par Hofmann et Dorda [10].

4) $\Delta V_{\mathrm{FB}}$ est fonction linéaire de $t_{\mathrm{ox}}^{2}$ (Fig. 10); cela permet d'affirmer (résolution et discussion de l'équation de Poisson) que cette dérive ne peut pas être la conséquence d'une distribution uniforme de la charge positive dans $\mathrm{SiO}_{2}$. Ce point est d'ailleurs confirmé par des mesures de photocourant faites par nous même

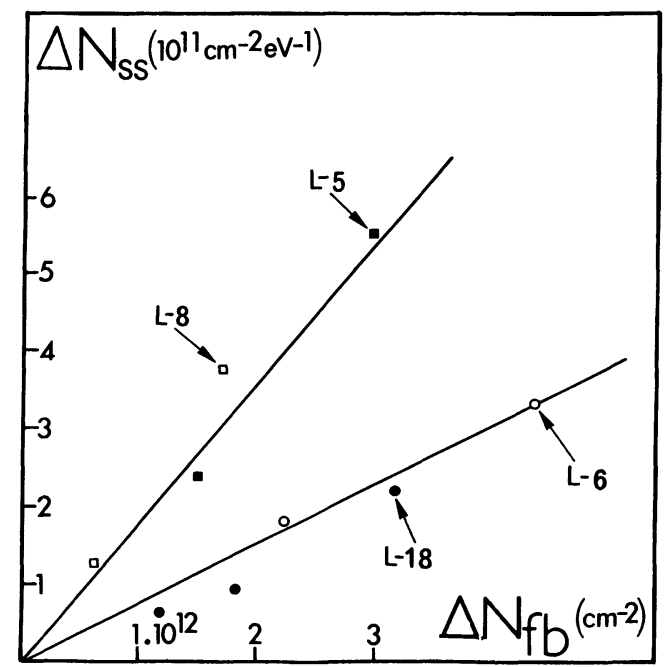

Fig. 9. - Variation de la densité d'états d'interface rapides $\Delta N_{\mathrm{ss}}$ en fonction de la densité de charges interfaciales $\Delta N_{\mathrm{FB}}$.

[Variations of the fast interface state density $N_{\mathrm{ss}}$ versus interfacial charge density $\Delta N_{\mathrm{FB}}$.] 


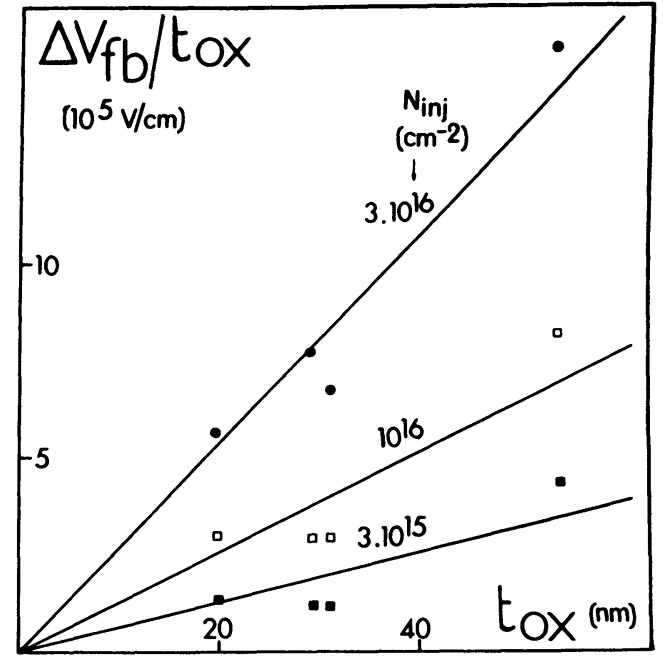

Fig. 10. - Courbes d'évolution de $\Delta V_{\mathrm{FB}} / t_{\mathrm{ox}}$ en fonction de $t_{\text {ox}}$.

[Evolution curves of $\Delta V_{\mathrm{FB}} / t_{\mathrm{ox}}$ versus $t_{\mathrm{ox}}$ ]

(non publiées) et par d'autres [22]. Mais elle indiquerait plutôt la diffusion d'une espèce $X$, à partir du volume vers l'interface $\mathrm{Si}_{-} \mathrm{SiO}_{2}$; ce qui favoriserait la formation des états lents, conformément au modèle proposé par Fischetti et al. [7], et la génération des états-pièges induits par l'injection. Par exemple, l'influence de l'eau suggère que les processus physico-chimiques sont essentiellement contrôlés par l'hydrogène [1720].

La présence d'électrons injectés favorise la dissociation de certains groupements, tels que $\mathrm{Si}-\mathrm{H}$ et $\mathrm{SiOH}$ (ou $\mathrm{AlH}$ ). Il en résulte ainsi la formation de nouveaux états de surface $[13,14]$ et d'atomes d'hydrogène interstitiels qui diffusent ensuite vers le volume de l'oxyde. Cette diffusion peut être influencée par la présence des électrons injectés. De plus, ils peuvent réagir avec des liaisons silicium ou oxygène ouvertes, formant ainsi des centres de capture [15].

Par ailleurs, la corrélation observée entre les charges présentes à proximité de l'interface injectante et le processus de claquage, suggère que celui-ci est initié dans la région de l'oxyde proche de la cathode. Comme l'a démontré Di Stefano [21], il ne s'agit pas d'un mécanisme semblable à un processus d'avalanche (création par impact ionisant de paires électron-trou). En fait, la formation d'une charge d'espace positive produit une modification importante du champ interne à l'oxyde. Cet accroissement entraîne une accélération des électrons injectés par mécanisme F.N. Ceux-ci deviennent alors suffisamment énergétiques pour provoquer la rupture de certaines liaisons (fragilisées par des contraintes). Ainsi l'effet simultané du champ et de l'injection est à l'origine du processus de claquage destructif.

\section{Conclusion.}

Nous avons décrit une technique permettant d'étudier la dégradation des structures MOS à oxyde mince (épaisseur inférieure à $20 \mathrm{~nm}$ ) quand elles sont soumises à une injection tunnel Fowler-Nordheim. Le principe consiste à suivre l'évolution de la polarisation appliquée à la grille, pendant une contrainte à courant (ou flux d'électrons injectés) constant. Sous injection, il y a remplissage des pièges natifs (densité variable selon la technologie entre $10^{11} \mathrm{~cm}^{-2}$ et $10^{13} \mathrm{~cm}^{-2}$ et section efficace de $10^{-15} \mathrm{~cm}^{2}$ et $10^{-20}$ $\mathrm{cm}^{2}$ ). Des centres nouveaux se forment pendant la contrainte, à proximité de l'interface cathodique avec un taux de $5 \times 10^{-8}$ et une section efficace de $10^{-20}$ $\mathrm{cm}^{2}$ à $10^{-19} \mathrm{~cm}^{2}$. Le claquage destructif des oxydes se produit après l'injection d'une charge de 15 à $30 \mathrm{C} / \mathrm{cm}^{2}$ (selon la technologie). L'expérience montre que toutes ces caractéristiques du piégeage dans les structures MOS à oxyde mince dépendent de l'épaisseur de $\mathrm{SiO}_{2}$, de la concentration et de la nature de l'impureté de dopage dans la grille Si-Poly et dans le substrat.

L'évolution $\Delta V_{\mathrm{R}}$ de la tension à injection constante peut être comparée à celle de la tension de commande des structures mémoires, au cours des cycles programmation-effacement. La méthode proposée et les résultats présentés peuvent donc avoir une application directe en ce qui concerne la compréhension de la dégradation de telles structures.

\section{Annexe.}

Nous présentons dans cette annexe le fondement théorique de la procédure expérimentale, qui nous a permis de mettre en évidence la création de nouveaux pièges dans le volume de l'oxyde pendant l'injection tunnel Fowler-Nordheim. Les cinétiques de variation de la population des états-pièges dans les couches d'oxyde thermique sont généralement décrites par des équations du premier ordre [1] :

$$
\frac{\partial N_{\mathrm{t}}}{\partial t}=\sigma\left\{N_{\mathrm{t}}(\infty)-N_{\mathrm{t}}(t)\right\} \cdot \frac{J}{q} \text { avec } \frac{J}{q}=\frac{N_{\mathrm{inj}}}{t_{\mathrm{inj}}}
$$

$N_{\mathrm{t}}$ est la densité de pièges occupés dans l'oxyde, $N_{\text {inj }}$ est le nombre de porteurs injectés pendant le temps de contrainte,

$t_{\text {inj }}$ (dans le cas présent, l'injection est réalisée à courant constant).

En supposant que $\sigma$ ne varie pas (température et champ fixes), l'intégration de (A.1) conduit à :

$$
N_{\mathrm{t}}\left(\sigma, t_{\mathrm{inj}}\right)=N_{\mathrm{t}}(\infty)\left\{1-\exp \left(-\frac{\sigma J}{q} t_{\mathrm{inj}}\right)\right\} .
$$


La dérive en tension des caractéristiques électriques (par exemple condition de champ constant à la cathode) est alors :

$$
\Delta V_{\mathrm{G}}=\Delta V_{\mathrm{R}}=\frac{q}{\varepsilon_{\mathrm{ox}}} \bar{x} N_{\mathrm{t}}(\infty)\left\{1-\exp \left(-\frac{\sigma J}{q} t_{\mathrm{inj}}\right)\right\}
$$

où $\bar{x}$ est le barycentre des pièges positionné par rapport à l'anode [1]. En différentiant cette expression, on obtient l'efficacité de piégeage :

$$
\frac{\mathrm{d}\left(\Delta V_{\mathrm{G}}\right)}{\mathrm{d} t_{\mathrm{inj}}}=\frac{q}{\varepsilon_{\mathrm{ox}}} \bar{x} N_{\mathrm{t}}(\infty) \sigma \cdot \exp \left(-\frac{\sigma J}{q} t_{\mathrm{inj}}\right)=\frac{1}{C_{\mathrm{ox}}} \eta_{\mathrm{eff}} .
$$

Il est évident que $\Delta V_{\mathrm{G}}$ en fonction de $t_{\mathrm{inj}}$ doit saturer si $N_{\mathrm{inj}}=J t_{\mathrm{inj}}$ est suffisamment grand et que $\ln \left(\mathrm{d} \Delta V_{\mathrm{G}} / \mathrm{d} t_{\mathrm{inj}}\right)$ en fonction de $t_{\mathrm{inj}}$ devrait présenter une décroissance linéaire. Ce n'est pas le cas, pour les couches d'oxyde très minces (courbes de la Fig. 2). Cette évolution est due à la création de pièges pendant l'injection tunnel, avec un taux de création $r$ défini $\operatorname{par} \partial N_{\text {ind }} / \partial N_{\text {inj }}$. Le nombre total des pièges présents augmente pendant l'injection; il faut alors modifier les équations (A.1-A.4). Notons par $N_{\text {ind }}(t)$ le nombre de centres induits occupés à un instant $t$. Entre les instants 0 et $\delta t$, il y a création de $\delta N_{\text {ind }}$ pièges dont la cinétique de remplissage est décrite par (A.1) soit :

$$
\frac{\partial}{\partial t}\left(\partial N_{\text {ind }}\right)=\sigma_{\text {ind }} \cdot \frac{J}{q}\left\{\delta N_{\text {ind }}(0)-\delta N_{\text {ind }}(t)\right\} .
$$

L'intégration sur un intervalle de temps $\delta t$, soit de 0 à $t=\delta t$ (ou de $t$ à $t+\delta t)$ conduit à :

$$
\delta N_{\text {ind }}=\delta N_{\text {ind }}(0)-\delta N_{\text {ind }}(0) \exp \left(-\frac{\sigma_{\text {ind }} J}{q} t\right)
$$

où $\delta N_{\text {ind }}(0)$ est le nombre total de centres induits (occupés ou vides); en considérant que le taux de création est constant, on obtient :

$$
\frac{\delta N_{\text {ind }}}{\delta t}=r \cdot \frac{J}{q}\left\{1-\exp \left(-\frac{\sigma_{\text {ind }} J}{q} t\right)\right\}
$$

soit en intégrant sur toute la durée de l'injection de 0 à $t_{\text {inj }}$,

$$
\begin{aligned}
N_{\text {ind }}\left(t_{\text {inj }}\right)=r & \frac{J}{q} t_{\text {inj }}-\frac{1}{\sigma_{\text {ind }}} r \frac{J}{q} \times \\
& \times\left\{1-\exp \left(-\frac{\sigma_{\text {ind }} J}{q} t_{\text {inj }}\right)\right\} .
\end{aligned}
$$

La dérive de $V_{R}$ doit être explicitée en tenant compte de tous les centres chargés,

$$
\begin{aligned}
\Delta V_{\mathbf{R}}\left(t_{\mathrm{inj}}\right)= & \frac{q}{\varepsilon_{\mathrm{ox}}} \bar{x}_{\mathrm{ex}} N_{\mathrm{ex}}(\infty)\left\{1-\exp \left(-\frac{\sigma_{\mathrm{ex}} J}{q} t_{\mathrm{inj}}\right)\right\}+ \\
+ & \frac{q}{\varepsilon_{\mathrm{ox}}} \bar{x}_{\mathrm{ind}}\left(r \frac{J}{q} t_{\mathrm{inj}}-r \frac{J}{q} \frac{1}{\sigma_{\mathrm{ind}}} \times\right. \\
& \left.\times\left\{1-\exp \left(-\frac{\sigma_{\mathrm{ind}} J}{q}\right) t_{\mathrm{inj}}\right\}\right) .
\end{aligned}
$$

\section{Bibliographie}

[1] Dimaria, D. J., Proc. Intern' 1 topical conf. on Physics of $\mathrm{SiO}_{2}$ and its interfaces, Yorktown Heights, New York (1978) p. 160.

[2] Balland, B., Defects centres in $\mathrm{SiO}_{2}:$ Carriers Trapping and Detrapping in Instabilities in Integrated Devices. Edited by G. Barbottin and A. Vapaille (North-Holland public.) 1985.

[3] Young, D. R., Insulating Films on Semiconductors 1979. Edited by G. G. Roberts and M. J. Morant (The Institute of Physics, London) 1980, p. 28.

[4] Feigl, F. J., Young, D. R., Dimaria, D. J. and LAI, S. K., in Insulating Films on Semiconductors, Edited by M. Schultz and G. Pensel (SpringerVerlag, Berlin) 1981, p. 104.

[5] LaI, S. K., YounG, D. R., in Insulating Films on Semiconductors, Edited by M. Schultz and G. Pensel (Springer-Verlag, Berlin) 1981, p. 118.

[6] Dekeersmaecker, R. F., ESSDERC'83, 8th Symposium on Solid State Device Technology, Canterbury (1983).
[7] Fischetti, M. V., Gastaldi, R., MagGioni, F., Modelli, A., J. Appl. Phys. 53 (1982).

[8] Harari, E., J. Appl. Phys. 49 (1978) 2478.

[9] Solomon, P., J. Appl. Phys. 48 (1977) 3843.

[10] Hofmann, K. R., Dorda, G., in Insulating Films on Semiconductors, Edited by M. Schultz and G. Pensel (Springer-Verlag, Berlin) 1981, p. 122.

[11] Dekeersmaecker, R. F., Insulating Films on Semiconductors, Ed. J. F. Verweij and D. R. Wolters, Proceedings of the International Conference INFOS 83, Eindhoven, The Netherlands (1983).

[12] Balk, P., Inst. Phys. Conf. Ser. Nr 69, Proceedings of ESSDERC/SSSDT'83, Canterbury (1983).

[13] Plossu, C., Balland, B., Cambou, B., ESSDERC'83, 8th Symposium on Solid State Device Technology, Canterbury (1983).

[14] Balland, B., Plossu, C., Pinard, P. and Borel, J., Extended Abstracts of the 16th 1984 International Conference on Solid State Device and Materials, Kobe (1984) pp. 103-106. 
[15] Horiguchi, S., Kobayaschi, T. and Saito, H., Extended Abstracts of the 16th International Conference on Solid State Device and Materials, Kobe (1984) pp. 99-102.

[16] Nicollian, E. H. and Brews, J. A., MOS Physics and Technology (John Wiley, New York) 1982.

[17] Irene, E. A., The Physics of $\mathrm{SiO}_{2}$ and its interfaces, New York (Pantelides Pergamon Press) 1978, p. 205.
[18] Liang, M. S., Hu, C., International Electron Devices Meeting (IEEE), Washington (1981).

[19] Nicollian, E. H., Berglund, C. N., J. Appl. Phys. 41 (1970) 3025.

[20] Dimaria, D. J., J. Appl. Phys. 52 (1981) 7251.

[21] Distefano, T. H., ShatzKes, M., J. Vac. Sci. Technol. 12 (1) (1975) 37.

[22] Heyns, M. M., Dekeersmaecker, R. F. and HilLEN, M. W., Appl. Phys. Lett. 44 (2) (1984) 202. 